\title{
Molecular theory of partial molar volume and its applications to biomolecular systems
}

\author{
T.Imai \\ Department of Bioscience and Bioinformatics, Ritsumeikan University, Kusatsu, Shiga 525-8577, Japan
}

Received May 3, 2007, in final form June 8, 2007

\begin{abstract}
The partial molar volume (PMV) is a thermodynamic quantity which contains important information about the solute-solvent interactions as well as the solute structure in solution. Additionally, the PMV is the most essential quantity in the analysis of the pressure effect on chemical reactions. This article reviews the recent developments in molecular theories of the PMV, especially the reference interaction site model (RISM) theory of molecular liquids and its three-dimensional generalization version (3D-RISM), which are combined with the Kirkwood-Buff solution theory to calculate the PMV. This article also introduces our recent applications of the theory to some interesting issues concerning the PMV of biomolecules. In addition, theoretical representations of the effects of intramolecular fluctuation on the PMV, which are significant for biomacromolecules, are briefly discussed.
\end{abstract}

Key words: partial molar volume, pressure effect, biomolecular solution, Kirkwood-Buff theory, RISM theory

PACS: 05.20.Jj, 05.70.Ce, 62.50.+p, 65.20.+w, 82.60.Lf, 87.15.Aa

\section{Introduction}

\subsection{General description of partial molar volume}

The partial molar volume (PMV) of a solute in solution $\bar{V}_{\mathrm{u}}$ is defined as the volume change of solution when the solute is immersed into the solution:

$$
\bar{V}_{\mathrm{u}}=\left(\frac{\partial V}{\partial N_{\mathrm{u}}}\right)_{T, p, N_{\mathrm{v}}},
$$

where $V, T$, and $p$ are respectively the volume, temperature, and pressure of the solution system, and $N_{\mathrm{u}}$ and $N_{\mathrm{v}}$ are the numbers of solutes and solvents, respectively. This definition actually corresponds to the Archimedes' way in the third century B.C.: he immersed the king's crown into a container full of water and measured the volume of the overflow to determine the volume of the crown of complex shape. Thus, the PMV is a thermodynamic quantity primarily representing the "intrinsic volume" of the solute. From the microscopic viewpoint at the molecular level, however, the PMV is also affected by the solute-solvent interaction or solvation. For a typical example, lithium ion has a negative PMV. In fact, this strange "negative volume" is a clear manifestation of the strong electrostatic interaction between the ion and water, which is the so-called electrostriction [1]. The PMV thus includes important information on the solute-solvent interaction in solution.

According to thermodynamics, the PMV is also expressed by the pressure derivative of the chemical potential of solute $\mu_{\mathrm{u}}$ :

$$
\bar{V}_{\mathrm{u}}=\left(\frac{\partial \mu_{\mathrm{u}}}{\partial p}\right)_{T} .
$$

In this aspect, the PMV is the most essential quantity in the analysis of the pressure effect on chemical reactions. The pressure dependence of a chemical equilibrium constant $K$ is determined by the thermodynamic relation [2]:

$$
\left(\frac{\partial \ln K}{\partial p}\right)_{T}=-\frac{\Delta \bar{V}}{R T},
$$


where $\Delta \bar{V}$ is the reaction volume, that is, the PMV difference between reactant and product. This is an expression of Le Châtelier's principle. When pressure is applied to the system, the equilibrium shifts to the state with smaller PMV. As an interesting example, here let us consider the pressureinduced denaturation of protein. It has been well known in the fields of biochemistry and biophysics that a protein is denatured or unfolded by applying pressure. It follows from equation (3) that the PMV of the denatured structure is smaller than that of the native structure. Therefore, the question "why and how pressure changes the structure of a protein" can be converted into "why and how the PMV of the denatured structure of the protein is smaller than that of the native structure" under the two-state assumption of the native-denatured equilibrium. In addition, if the chemical reaction is to be described by the transition state theory, even the pressure dependence of the reaction rate constant $k$ can be predicted by the PMV difference between reactant and the transition state $\Delta \bar{V}^{\ddagger}$, namely the activation volume [3]:

$$
\left(\frac{\partial \ln k}{\partial p}\right)_{T}=-\frac{\Delta \bar{V}^{\ddagger}}{R T} .
$$

In this way, the PMV plays an essential role in analyzing the pressure effect on chemical reactions in solution.

\subsection{Historical background}

There is a large volume of experimental work devoted to the PMV and the reaction volume of various molecules, such as ions [1], organic compounds [4,5], biomolecules [5-7], and proteins $[8,9]$, in solution. Development of theories for calculating the PMV, however, had lagged behind the experimental studies until recently. If the geometric volumes such as the van der Waals volume and the so-called molecular volume could perform the role of the PMV, there would be no difficulty, because the geometric volume calculation based on the atomic diameters defined in an appropriate manner has been established [10-13]. However, the PMV cannot be replaced by such a geometric volume because it is a thermodynamic quantity. Considering that the PMV is actually expressed by the pressure derivative of the chemical potential of solute, the calculation of PMV should be as difficult as the free energy calculation.

In order to avoid the direct calculation, some empirical methods have been proposed [5-7,14,15]. In the empirical approaches, most strategies are to decompose the PMV experimentally obtained for various molecules into several contributions intuitively, and to reconstruct the PMV of a desired molecule by a superposition of those contributions. These methods are essentially based on the Traube additive scheme [16] proposed in the nineteenth century. Although the empirical methods have apparently succeed in predicting the PMV itself, they have obvious difficulty in the application to the volume change, for example, upon the conformational transition, which should involve more subtle effects of solvation. That is a fatal drawback because we are generally more interested in the volume change than the PMV itself. The most significant problem is that such empirical methods are basically incapable of providing a molecular picture of the PMV as a thermodynamic quantity.

There are mainly two theoretical approaches based on statistical mechanics of liquids to the calculation of the PMV as a real thermodynamic quantity. One of them is the scaled particle theory (SPT) [17-19]. The SPT yields an approximate expression for the reversible work necessary for introducing a spherical particle into a fluid, which is the so-called free energy of cavity formation. Hirata and Arakawa [20] obtained an analytical expression for the PMV of cavity formation, or the so-called intrinsic volume, by taking the pressure derivative of the cavity formation free energy, and succeeded in extracting the intrinsic volume of ions in aqueous solution. The same or similar analytical equations have been applied to the analysis of the PMV in some solution systems [21-27]. However, the SPT has two essential difficulties in its application to the systems of chemical and biochemical interest. First, the application of the original SPT is limited to spherical solute only or requires an unreasonable transformation from the molecule of nonspherical shape to a spherical particle. The other difficulty is that the SPT is incapable of calculating the electrostatic effects on the PMV such as electrostriction within its theoretical framework. Irisa, Nagayama, and Hirata [28] resolved the former problem by extending the theory to arbitrary shaped solutes. Then they 
succeeded in calculating the PMV of molecular solutes by using the extended SPT [29]. However, the latter problem remains substantially unresolved, even though the combination with dielectric continuum models was proposed [25,30]. Thus, the SPT is useful for calculating the PMV only of the systems in which the solvent-exclusion effect is significant rather than the solute-solvent electrostatic interaction.

In addition, analytical equations for the reaction volume of the contact-complex formation of two hard-spheres were also derived from analytical formulae of the cavity distribution function $[31,32]$. However, these approaches are faced with essentially the same difficulties as the SPT in the application to more complicated molecular systems.

The other approach is based on the Kirkwood-Buff (KB) solution theory [33]. The theory provides a general framework for evaluating thermodynamic quantities, including the PMV, of a liquid mixture in terms of the density pair correlation functions. In the KB theory, the PMV of a solute is given by

$$
\bar{V}=k_{\mathrm{B}} T \chi_{\mathrm{T}}-\int_{0}^{\infty}(g(r)-1) 4 \pi r^{2} \mathrm{~d} r
$$

where $\chi_{\mathrm{T}}$ is the isothermal compressibility of solution, and $g(r)$ is the radial distribution function (RDF) between the solute and solvent. If the RDF has been obtained, the PMV can be readily calculated through this equation. Therefore, the problem is how to obtain the RDF.

Molecular simulation may be a possible candidate to produce the RDF. However, attempts to combine the KB theory with molecular simulation are still limited to small molecules [34-38]. Another approach is to employ molecular liquid theories [39,40], such as the reference hypernetted chain (RHNC) [41] and the reference interaction site model (RISM) [42,43] theories, which yield the RDF through statistical-mechanical integral equations. The RHNC theory coupled with the KB theory was applied to the PMV of ions in aqueous solution [44,45]. However, practically the RHNC theory which uses the rotational-invariant expansion method may not be used for solutes of complex shape like biomolecules. The RISM theory which adopts the site-site description of the correlation functions can be applied to molecules with arbitrary geometry. Especially since Hirata et al. [46-48] extended the RISM theory to molecules with charged sites by a renormalization of the Coulomb potentials, the RISM theory has been applied to a wide variety of molecular systems $[40,49]$. By combination with the KB theory, the RISM theory has succeeded in calculating and analyzing the PMV for various molecular systems [29]: ions [50,51], hydrocarbons [52-54], and biomolecules [55-57] in aqueous solution as well as other nonaqueous solution systems [58-60]. More recently, the RISM theory was generalized to describe the three-dimensional spatial correlation functions of solvent around a solute molecule $[40,61,62]$. The theory, so-called 3D-RISM theory, was also combined with the KB theory and has been successfully applied to the PMV, especially of biomolecules such as amino acids [63], peptides [63-65], and proteins [66-71]. Thus, the RISM theory is the most successful and promising method of investigating the PMV as well as other thermodynamic properties in molecular solution systems.

\subsection{Outline of this review}

The remainder of this article is organized as follows. Section 2 summarizes the RISM and 3DRISM theories and the PMV calculation using the theories in combination with the KB theory. The quantitative capabilities of some different approximations adopted in the theories are evaluated for the PMV of amino acids as instances of multiatomic molecules. Section 3 introduces our recent applications of the 3D-RISM theory to the PMV of biomolecules, especially proteins. It is shown that the PMV of proteins can be efficiently analyzed by a theoretical method of volume decomposition. As interesting examples of the volume decomposition analysis, recent studies on the PMV changes associated with the conformational transition of a protein and with ligand-protein binding are briefly introduced. Section 4 presents the theoretical description of the effects of intramolecular fluctuation on the PMV. The effects are particularly significant in analyzing the volumetric properties of polyatomic biomolecules which should considerably fluctuate in solution. Section 5 concludes this review with some remarks. 


\section{Molecular theory of partial molar volume}

\subsection{Partial molar volume calculation based on the RISM theory}

\subsubsection{RISM theory}

The RISM integral equation defines the relation between the site-site total and direct correlation functions, $h_{\alpha \gamma}(r)$ and $c_{\alpha \gamma}(r)[39,40,42,43]$ :

$$
h_{\alpha \gamma}(r)=\sum_{\eta, \nu} \int \mathrm{d} \mathbf{r}^{\prime} \int \mathrm{d} \mathbf{r}^{\prime \prime} w_{\alpha \eta}\left(r^{\prime}\right) c_{\eta \nu}\left(\left|\mathbf{r}^{\prime}-\mathbf{r}^{\prime \prime}\right|\right)\left(w_{\nu \gamma}\left(\left|\mathbf{r}^{\prime \prime}-\mathbf{r}\right|\right)+\rho_{\nu} h_{\nu \gamma}\left(\left|\mathbf{r}^{\prime \prime}-\mathbf{r}\right|\right)\right),
$$

where $w_{\alpha \gamma}(r)$ is the intramolecular correlation function, which determines the distances of site pairs, namely the geometry of each molecular species, and $\rho_{\nu}$ is the number density of the species. The site-site total correlation function is related to the site-site RDF by $h_{\alpha \gamma}(r)=g_{\alpha \gamma}(r)-1$. The RISM equation is generally coupled with the closure equation used for simple liquids:

$$
h_{\alpha \gamma}(r)=\exp \left(-\beta u_{\alpha \gamma}(r)+h_{\alpha \gamma}(r)-c_{\alpha \gamma}(r)+b_{\alpha \gamma}(r)\right)-1,
$$

where $\beta=1 /\left(k_{\mathrm{B}} T\right)$ is the inverse of the Boltzmann constant times temperature, $u_{\alpha \gamma}(r)$ is the pair potential function, and $b_{\alpha \gamma}(r)$ is the bridge function, which determines the approximation of the closure equation. If the approximation $b_{\alpha \gamma}(r)=0$ is adopted, the equation corresponds to the hypernetted chain (HNC) equation [39,72], which is the most common one.

Although the RISM theory can yield the correlation functions of a multi-component solution under an arbitrary condition, here let us consider a two-component (solute and solvent) system at infinite dilution because we usually deal with the PMV under that condition. In the twocomponent system, equation (6) can be decomposed into the solvent-solvent and solute-solvent equations $[40,48]$ :

$$
\begin{aligned}
& h_{\alpha \gamma}^{\mathrm{vv}}(r)=\sum_{\eta, \nu} \int \mathrm{d} \mathbf{r}^{\prime} \int \mathrm{d} \mathbf{r}^{\prime \prime} w_{\alpha \eta}^{\mathrm{vv}}\left(r^{\prime}\right) c_{\eta \nu}^{\mathrm{vv}}\left(\left|\mathbf{r}^{\prime}-\mathbf{r}^{\prime \prime}\right|\right)\left(w_{\nu \gamma}^{\mathrm{vv}}\left(\left|\mathbf{r}^{\prime \prime}-\mathbf{r}\right|\right)+\rho h_{\nu \gamma}^{\mathrm{vv}}\left(\left|\mathbf{r}^{\prime \prime}-\mathbf{r}\right|\right)\right), \\
& h_{\alpha \gamma}^{\mathrm{uv}}(r)=\sum_{\eta, \nu} \int \mathrm{d} \mathbf{r}^{\prime} \int \mathrm{d} \mathbf{r}^{\prime \prime} w_{\alpha \eta}^{\mathrm{uu}}\left(r^{\prime}\right) c_{\eta \nu}^{\mathrm{uv}}\left(\left|\mathbf{r}^{\prime}-\mathbf{r}^{\prime \prime}\right|\right)\left(w_{\nu \gamma}^{\mathrm{vv}}\left(\left|\mathbf{r}^{\prime \prime}-\mathbf{r}\right|\right)+\rho h_{\nu \gamma}^{\mathrm{vv}}\left(\left|\mathbf{r}^{\prime \prime}-\mathbf{r}\right|\right)\right),
\end{aligned}
$$

where superscripts "v" and "u" denote solvent and solute, respectively. Using these equations, we can obtain the solute-solvent correlation functions in the following way. First, we calculate the correlation functions for bulk solvent from the intramolecular correlations $w_{\alpha \gamma}^{\mathrm{vv}}(r)$ and the intermolecular potentials $u_{\alpha \gamma}^{\mathrm{vv}}(r)$ of the solvent as well as the thermodymanic conditions, $\rho$ and $T$, by iteratively solving equations (7) and (8). Then, we calculate the solute-solvent correlation functions from the obtained solvent susceptibility, $w_{\alpha \gamma}^{\mathrm{vv}}(r)+\rho h_{\alpha \gamma}^{\mathrm{vv}}(r)$, as well as the intramolecular correlations of the solute $w_{\alpha \gamma}^{\mathrm{uu}}(r)$, the solute-solvent intermolecular potentials $u_{\alpha \gamma}^{\mathrm{uv}}(r)$, and the temperature $T$ through the combination of equations (7) and (9). In practice, the convolution integrals in equations (8) and (9) are calculated by the fast Fourier transform technique. The long-range electrostatic asymptotics are separated out and treated analytically.

\subsubsection{Site-site Kirkwood-Buff theory}

The KB equation (5) has been extended to use the site-site correlation functions instead of the molecular ones $[50,55,58]$. The PMV at infinite dilution is calculated from the site-site total correlation function of an arbitrary solute-solvent site pair or from all of the site-site direct correlation functions:

$$
\begin{aligned}
\bar{V} & =k_{\mathrm{B}} T \chi_{\mathrm{T}}^{0}-\int_{0}^{\infty} h_{\alpha \gamma}^{\mathrm{uv}}(r) 4 \pi r^{2} \mathrm{~d} r \\
& =k_{\mathrm{B}} T \chi_{\mathrm{T}}^{0}\left(1-\rho \sum_{\alpha, \gamma} \int_{0}^{\infty} c_{\alpha \gamma}^{\mathrm{uv}}(r) 4 \pi r^{2} \mathrm{~d} r\right)
\end{aligned}
$$


where $\chi_{\mathrm{T}}^{0}$ is the isothermal compressibility of pure solvent. It is an advantage of using the latter equation that we can obtain each site contribution to the total PMV. In the equation, the total PMV is naturally expressed by the sum of the site contributions $\bar{V}_{\alpha}$ :

$$
\begin{aligned}
\bar{V} & =k_{\mathrm{B}} T \chi_{\mathrm{T}}^{0}+\sum_{\alpha} \bar{V}_{\alpha} \\
\bar{V}_{\alpha} & =-\rho k_{\mathrm{B}} T \chi_{\mathrm{T}}^{0} \sum_{\gamma} \int_{0}^{\infty} c_{\alpha \gamma}^{\mathrm{uv}}(r) 4 \pi r^{2} \mathrm{~d} r
\end{aligned}
$$

where $k_{\mathrm{B}} T \chi_{\mathrm{T}}^{0}$ is the ideal contribution only from the translational degrees of freedom of the solute molecule and therefore cannot be divided into the site contributions.

The isothermal compressibility can also be calculated from the site-site direct correlation functions of solvent [55],

$$
\chi_{\mathrm{T}}^{0}=\left(\rho k_{\mathrm{B}} T\left(1-\rho \sum_{\alpha, \gamma} \int_{0}^{\infty} c_{\alpha \gamma}^{\mathrm{vv}}(r) 4 \pi r^{2} \mathrm{~d} r\right)\right)^{-1},
$$

instead of the usual compressibility equation.

\subsubsection{Numerical results for the partial molar volume of amino acids}

Figure 1(a) shows the comparison of the numerical results of the RISM theory with the experimental data for the PMV of twenty amino acids. In these calculations, the solute-solvent RISM equation (9) was coupled with several different closure approximations (7) for a comprehensive evaluation of the theory, while the dielectrically consistent RISM (DRISM) theory [73,74] was employed for obtaining the solvent-solvent correlation functions in common. As the intermolecular potentials, the Amber99 parameter set [75] and the SPC/E model [76] were used for amino acids and water, respectively.

It is apparent from the figure that the RISM equation coupled with the HNC closure, which adopts $b_{\alpha \gamma}(r)=0$ in equation (7), systematically underestimates the PMV, though the theoretical values show a linear correlation with the experimental data [55]. Based on further careful analysis and consideration, we found out that the linear correlation is attributed to reasonable description of the solute-solvent interactions by the theory, while the systematic deviation is ascribed to imperfect account of the solvent exclusion due to partial neglect of the multi-site correlation effects in the site-site treatment.

Recently, Kovalenko and Hirata [77] proposed an alternative closure, which is referred to as the $\mathrm{KH}$ closure, to prevent the artificial divergence sometimes occurring in the HNC closure for strongly associating molecular liquid mixtures. The KH closure combines the HNC approximation in the spatial regions of density depletion and the mean spherical approximation (MSA), $c_{\alpha \gamma}(r)=$ $-\beta u_{\alpha \gamma}(r)$, in the enrichment regions. The bridge function for the KH closure is given by

$$
b_{\alpha \gamma}(r)=\left\{\begin{array}{lll}
0, & \text { for } & d_{\alpha \gamma}(r)<0 \\
-d_{\alpha \gamma}(r)+\ln \left(1+d_{\alpha \gamma}(r)\right), & \text { for } & d_{\alpha \gamma}(r)>0
\end{array}\right.
$$

where $d_{\alpha \gamma}(r)=-\beta u_{\alpha \gamma}(r)+h_{\alpha \gamma}(r)-c_{\alpha \gamma}(r)$. Figure 1(a) demonstrates that the PMV values calculated by the RISM/KH theory are slightly larger than those of the RISM/HNC theory, though they are still underestimated [78]. This apparent improvement is due to the suppression of the solute-solvent association, which reduces the PMV, rather than the essential improvement in the description of the solvent exclusion. In general, a PMV value calculated by the RISM/KH theory is equivalent to or slightly larger than that of the RISM/HNC theory.

More recently, Kinoshita et al. [56] proposed an efficient bridge function to improve the theoretical estimation of PMV. The bridge function is based on what was originally developed for 
(a)

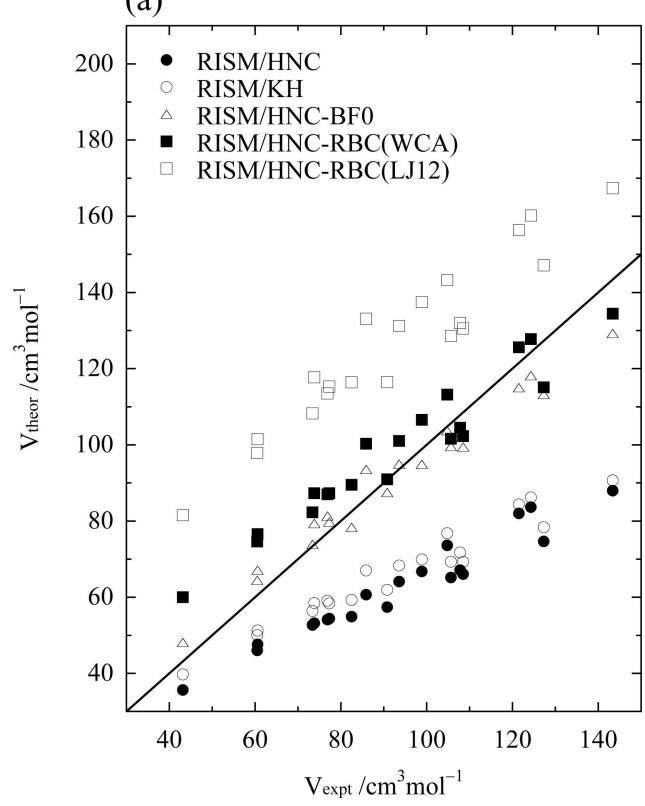

(b)

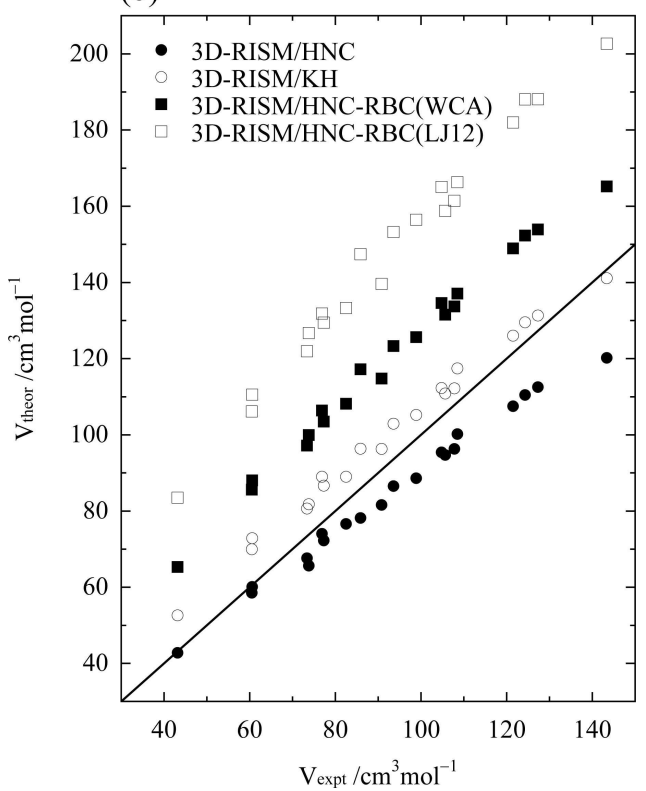

Figure 1. Partial molar volume of twenty amino acids calculated (a) by the combinations of the RISM equation with the five different closure approximations and (b) by the combinations of the 3D-RISM equation with the four different closure approximations, plotted against the corresponding experimental data. The solid line marks the exact coincidence. New data recalculated using the Amber99 parameter set instead of the older one employed in our previous studies $[55,56,63]$ are shown here.

hard-sphere liquids to improve the description of liquid structure [79] and was modified to be used for polar molecules including charged sites. The site-site bridge function is given by

$$
b_{\alpha \gamma}(r)=\left\{\begin{array}{lll}
\frac{-\frac{1}{2}\left(h_{\alpha \gamma}^{(0)}(r)-c_{\alpha \gamma}^{(0)}(r)\right)^{2}}{1+\frac{4}{5}\left(h_{\alpha \gamma}^{(0)}(r)-c_{\alpha \gamma}^{(0)}(r)\right)} & \text { for } \quad h_{\alpha \gamma}^{(0)}(r)-c_{\alpha \gamma}^{(0)}(r)>0, \\
-\frac{1}{2}\left(h_{\alpha \gamma}^{(0)}(r)-c_{\alpha \gamma}^{(0)}(r)\right)^{2} & \text { for } \quad h_{\alpha \gamma}^{(0)}(r)-c_{\alpha \gamma}^{(0)}(r)<0,
\end{array}\right.
$$

where the superscript "(0)" denotes that the function is calculated for the molecule whose site charges are all switched off. The closure including this bridge function is referred to as the HNC-BF0 closure. As seen in figure 1(a), the use of the HNC-BF0 closure drastically improves the agreement between the calculated values and the experimental data, in spite of the fact that the bridge function does not directly incorporate the multi-site correlation effects neglected in the site-site HNC approximation which cause the substantial underestimation. However, the RISM/HNC-BF0 theory still underestimates the PMV of larger molecules with buried interaction sites because the multi-site correlation effects become more significant for such molecules, even though the estimation is much better than that of the RISM/HNC theory [56].

Kovalenko and Hirata [80] proposed another bridge function, originally in order to improve the theoretical estimation of the solvation free energy. The bridge function modifies the solute-solvent site-site correlations by adding some averaged short-range exclusion effect coming from the other solvent sites. It thus supplies some effect of the missing multi-site correlations. The bridge function takes the form:

$$
b_{\alpha \gamma}^{\mathrm{uv}}(r)=\sum_{\eta \neq \gamma} \ln \left(\int \mathrm{d} \mathbf{r}^{\prime} \exp \left(-\beta u_{\alpha \eta}^{\mathrm{uv}(\mathrm{r})}\left(r^{\prime}\right)\right) w_{\eta \gamma}^{\mathrm{vv}}\left(\left|\mathbf{r}^{\prime}-\mathbf{r}\right|\right)\right),
$$

where the superscript "(r)" denotes a short-range repulsion of the solute-solvent potential. This 
bridge correction is referred to as the repulsive bridge correction (RBC). When we are using the 12-6 Lennard-Jones (LJ) potential function, we may adopt the LJ $r^{-12}$ repulsive term or the Weeks-Chandler-Andersen (WCA) short-range repulsive term [81] as the short-range repulsion. It was found that the former gave better estimations for the solvation free energy than the latter [80]. However, figure 1(a) shows that the RBC using the LJ-12 repulsive term considerably overestimates the PMV in the whole range [78]. The RBC with the WCA repulsion provides more reasonable estimations, though it also overestimates for the smaller molecules. This result implies that the $\mathrm{RBC}$ is overabundant in the additional exclusion effect on the PMV.

In addition, Leu and Blankschtein [52] investigated the PMV as well as the structural and some other thermodynamic properties of hydrocarbons in aqueous solution by using closure approximations other than described above. They found that the quantitative predictive capabilities of the RISM theory were improved by adopting the Martynov-Sarkisov (MaS) closure [82] or its generalization, the Ballone-Pastore-Galli-Gazzillo (BPGG) closure [83]. The bridge function for the latter is given by

$$
b_{\alpha \gamma}(r)=\left(1+s\left(h_{\alpha \gamma}(r)-c_{\alpha \gamma}(r)\right)\right)^{1 / s}-\left(1+\left(h_{\alpha \gamma}(r)-c_{\alpha \gamma}(r)\right)\right),
$$

where $s$ is an adjustable parameter. When $s=1$, it reduces to the HNC closure, and when $s=2$, it corresponds to the MaS closure. They found that $s=1.79,1.96$, and 2.05 were the best choices for reproducing the thermodynamic properties of methane, ethane, and propane, respectively. However, this closure has not been examined for larger as well as polar molecules. Even if such examinations are done, the ad hoc determination of the adjustable parameter, which has no physical meaning, can make little physical sense.

In conclusion, when we employ the RISM theory to calculate the PMV, we should select an appropriate closure approximation. For a small molecule, typically which consists of less than ten atomic sites, we may adopt the $\mathrm{HNC}$ or $\mathrm{KH}$ closure as well as the HNC-BF0. For a molecule of moderate size, such as an amino acid, the HNC-BF0 closure is the best choice at the present stage of theoretical development. However, the site-site treatment of the RISM theory would fail to completely describe the buried sites in larger molecules, such as oligo-peptides and proteins. In such cases, we should employ a further developed theoretical treatment, namely the 3D-RISM theory, which will be introduced in the next subsection.

\subsubsection{Remark on the partial molar volume of ionic species}

For a neutral molecule, the PMV values calculated from the two different expressions of the site-site KB equation, equations (10) and (11), are identical to each other. However, the PMV value for an ionic molecule calculated from equation $(10), \bar{V}^{(h)}$, is theoretically different from that calculated from equation (11), $\bar{V}^{(c)}$, by a product of the volumetric properties of solvent and a term $D: \bar{V}^{(h)}=\bar{V}^{(c)}-\rho k_{\mathrm{B}} T \chi_{\mathrm{T}}^{0} D[45,50] . D$ is determined by the net charge of the ionic solute and the dielectric and thermodynamic properties of the pure solvent, but is not dependent on the molecular properties of the solute, such as charge distribution. Therefore, for ions whose charges are equal in magnitude but opposite in sign, the relation $D_{+}=-D_{-}$holds and $D$ does not contribute to the PMV of the salt. For example, $D$ of a monovalent cation in ambient water calculated based on the RISM/HNC theory is $-2.2 \mathrm{~cm}^{3} \mathrm{~mol}^{-1}[51]$.

Most experiments determine the PMV of salt and separate it into the individual ion contributions generally based on empirical assumptions which are not related to the term $D$. An experimental technique makes it possible to nonempirically determine the individual ion contributions based on the ultrasonic vibration potentials which are related to the PMV difference between the cation and anion [84]. It has been found that the PMV of an ion determined from the ultrasonic vibration potential measurement corresponds to $\bar{V}^{(c)}$ rather than $\bar{V}^{(h)}[45,85]$. Therefore, we usually calculate the PMV of ionic molecules by equation (11), regardless of whether or not the reference data are obtained from the ultrasonic vibration potential measurement. It should be noted that the discrepancy is not concerned with the PMV change associated with changes in the chemical properties of solute and with chemical reactions, such as the conformational change, unless the net charge of solute is varied. 


\subsection{Partial molar volume calculation based on the 3D-RISM theory}

\subsubsection{D-RISM theory}

The solute-solvent RISM equation has been generalized to describe the 3D spatial correlation functions of solvent around the solute $[40,61,62]$. The 3D-RISM equation is given by

$$
h_{\gamma}^{\mathrm{uv}}(\mathbf{r})=\sum_{\eta} \int \mathrm{d} \mathbf{r}^{\prime} c_{\eta}^{\mathrm{uv}}\left(\mathbf{r}^{\prime}\right)\left(w_{\eta \gamma}^{\mathrm{vv}}\left(\left|\mathbf{r}^{\prime}-\mathbf{r}\right|\right)+\rho h_{\eta \gamma}^{\mathrm{vv}}\left(\left|\mathbf{r}^{\prime}-\mathbf{r}\right|\right)\right)
$$

where $h_{\gamma}^{\mathrm{uv}}(\mathbf{r})$ and $c_{\gamma}^{\mathrm{uv}}(\mathbf{r})$ are, respectively, the 3D total and direct correlation functions of solvent site $\gamma$ around the solute. This equation is coupled with the $3 \mathrm{D}$ version of the closure equation:

$$
h_{\gamma}^{\mathrm{uv}}(\mathbf{r})=\exp \left(-\beta u_{\gamma}^{\mathrm{uv}}(\mathbf{r})+h_{\gamma}^{\mathrm{uv}}(\mathbf{r})-c_{\gamma}^{\mathrm{uv}}(\mathbf{r})+b_{\gamma}^{\mathrm{uv}}(\mathbf{r})\right)-1
$$

where $u_{\gamma}^{\text {uv }}(\mathbf{r})$ is the $3 \mathrm{D}$ interaction potential between solvent site $\gamma$ and the whole solute, which is generally obtained from the solute-solvent site-site potentials:

$$
u_{\gamma}^{\mathrm{uv}}(\mathbf{r})=\sum_{\alpha} u_{\alpha \gamma}^{\mathrm{uv}}\left(\left|\mathbf{r}_{\alpha}-\mathbf{r}\right|\right)
$$

where $\mathbf{r}_{\alpha}$ is the $3 \mathrm{D}$ coordinate of solute site $\alpha$.

In the 3D-RISM theory, we in advance obtain the site-site total correlation functions of solvent from a single-component RISM theory of pure solvent, equations (7) and (8). Then, we calculate the $3 \mathrm{D}$ correlation functions by the combination of equations (19) and (20) from the correlation functions of bulk solvent as well as the thermodynamic properties. The convolution integral in equation (19) is calculated by the 3D fast Fourier transform technique.

The 3D potential function may be calculated using the minimum image convention and the Ewald summation methods. In that case, the special electrostatic correlations for the 3D supercell finiteness are added to equation (20) in order to provide an accurate calculation for a solute containing site charges [86].

\subsubsection{D-RISM expression of the Kirkwood-Buff theory}

The KB equation (5) has also been generalized to the expression using the 3D correlation functions [63]:

$$
\begin{aligned}
& \bar{V}=k_{\mathrm{B}} T \chi_{\mathrm{T}}^{0}-\int h_{\gamma}^{\mathrm{uv}}(\mathbf{r}) \mathrm{d} \mathbf{r}, \\
& \bar{V}=k_{\mathrm{B}} T \chi_{\mathrm{T}}^{0}\left(1-\rho \sum_{\gamma} \int c_{\gamma}^{\mathrm{uv}}(\mathbf{r}) \mathrm{d} \mathbf{r}\right),
\end{aligned}
$$

where the isothermal compressibility $\chi_{\mathrm{T}}^{0}$ is calculated from the site-site correlation functions of solvent, as described in subsection 2.1.2. Equations (22) and (23) also give PMV values differing by a factor of $\rho k_{\mathrm{B}} T \chi_{\mathrm{T}}^{0} D$ for ionic species, as described in subsection 2.1.4.

Unlike the site-site KB equation (11), the 3D-KB equation cannot immediately provide the solute-site contributions to the PMV. The site contributions can be obtained through the RISM equation in the following way. First, we convert the $3 \mathrm{D}$ total correlation functions $h_{\gamma}^{\mathrm{uv}}(\mathbf{r})$ into the site-site total correlation functions $h_{\alpha \gamma}^{\mathrm{uv}}(r)$ by numerical averaging over the solute orientation. Second, we calculate the site-site direct correlation functions $c_{\alpha \gamma}^{\mathrm{uv}}(r)$ through the RISM equation (9). Finally, we obtain the site contributions from equation (13). This decomposition uses only the RISM ansatz (the decomposition of the molecular direct correlation function into a sum of additive contributions from interaction sites); it does not include the site-site closure approximation. 


\subsubsection{Numerical results for the partial molar volume of amino acids}

Figure 1(b) compares the PMV values of twenty amino acids calculated by the 3D-RISM theory with the corresponding experimental data. The 3D-RISM equation (19) was coupled with some closure approximations (20) again in order to evaluate the quantitative predictive capabilities of the theory. In the calculation, the potential parameters and the solvent correlation functions are the same as those used in the RISM calculation described above.

As seen in figure 1(b), the theoretical values of the 3D-RISM theory adopting the 3D-HNC approximation, $b_{\gamma}^{\mathrm{uv}}(\mathbf{r})=0$, are in quantitative agreement with the experimental data [63]. The drastic improvement as compared to the RISM/HNC theory is ascribed to the perfect description of the solvent-exclusion effect due to the solute. In the 3D-RISM theory, the solute sites are put in the $3 \mathrm{D}$ space; therefore, the solvent can never come into the solute repulsive core. The solvent-exclusion effect is an essential factor to quantitatively reproduce the PMV. As described above, the site-site treatment in the conventional RISM theory, especially under the $\mathrm{HNC}$ and $\mathrm{KH}$ approximation, underestimated the solvent-exclusion effect.

The ability of the other closure approximations has been also examined [78]. Figure 1(b) shows that the 3D-RISM/KH theory, in which the bridge function is given by the 3D version of equation (15), yields slightly larger PMV values compared to the 3D-RISM/HNC theory as in the case of the conventional RISM theory. The comparison with the experimental data indicates that the 3DRISM/KH theory is as practicable for the PMV calculation as the 3D-RISM/HNC theory. However, the 3D-RISM theory including the RBC, the 3D version of equation (17), overestimates the PMV, regardless of whether it adopts the WCA or LJ-12 repulsive term. The overestimation is more pronounced for the LJ-12 repulsion than for the WCA. The RBC is effective in the calculation of the solvation free energy, but not in the PMV calculation.

In conclusion, the 3D-RISM theory, especially when adopting the 3D-HNC or 3D-KH closure, is currently the most reliable and promising method for calculating the PMV of a solute molecule of complex shape with site charges. The theory has also been successfully applied to the calculation and analysis of the PMV of larger biomolecules such as proteins in aqueous solution. Some instances of the applications are introduced in the following section.

\section{Applications to the partial molar volume of proteins}

\subsection{Partial molar volume of proteins}

We have succeeded in calculating the PMV of several proteins by using the 3D-RISM/HNC theory in combination with the 3D-KB equation [66,67]. Figure 2 shows the comparison of the theoretical values with the corresponding experimental data. It is obvious that the theoretical results have a remarkable correlation with the experimental data. It was not until we employed the 3D-RISM theory that we had this almost perfect agreement with experimental data. In fact, the conventional RISM theory gave unphysically small, even negative, values of the PMV for peptides and proteins $[63,87,88]$.

The slight deviation between the theoretical and experimental values can be caused by some different factors. One is concerned with the intramolecular fluctuation of solute (protein) molecules. A protein molecule fluctuates in solution, and experiment observes the PMV of the fluctuating protein. On the other hand, this theory calculates the PMV of the protein fixed at the coordinates obtained from X-ray crystallography. The effect of intramolecular fluctuation on the PMV is discussed in the next section. The difference in the conditions of solution between the theory and each experiment might also influence the results. In experimental conditions, the solution contains protein molecules at a finite concentration. Moreover, some cosolvents, such as salt and buffer, are added to the solution. The theory ignores these effects. However, the effects of the self-association of protein and the protein-cosolvent interaction are generally insignificant with respect to the whole amount of the PMV [89]. Of course, some intrinsic imperfection in the potential function as well as the theory itself can cause the deviation. Nevertheless, our results demonstrate that those effects are only minor in considering the PMV of proteins. 


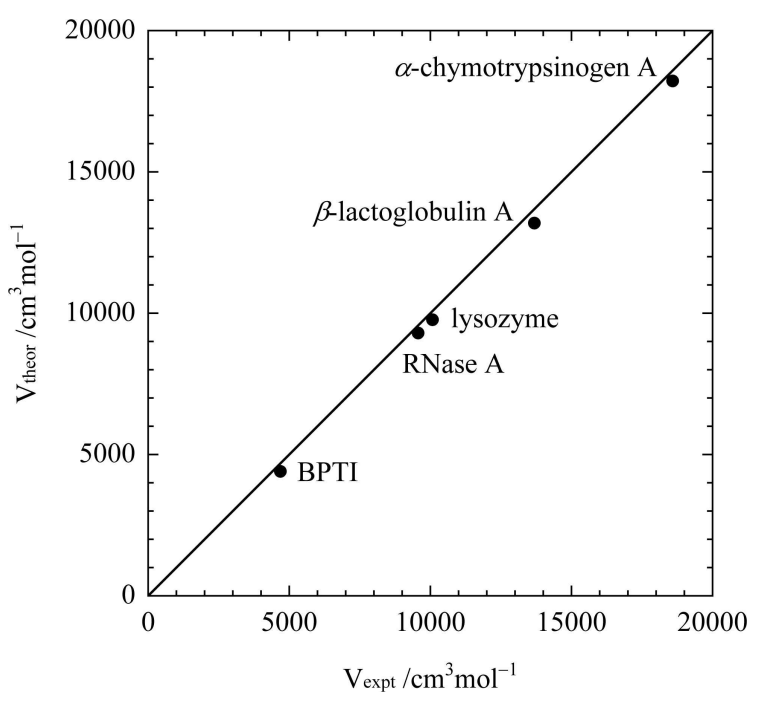

Figure 2. Partial molar volume of five representative proteins, bovine pancreatic trypsin inhibitor (BPTI), bovine pancreatic ribonuclease (RNase) A, hen egg-white lysozyme, bovine milk $\beta$ lactoglobulin A, and bovine pancreatic $\alpha$-chymotrypsinogen A, calculated by the $3 \mathrm{D}$-RISM/HNC theory, plotted against the corresponding experimental data. The solid line marks the exact coincidence.

\subsection{Volume decomposition analysis}

The PMV of a solute can be decomposed into the geometric volume of the solute itself, the so-called molecular volume $V_{\mathrm{M}}$, and the changes in the solvent volume caused by immersion of the solute, the so-called solvation volume $\Delta V_{\mathrm{s}}[7,90,91]$ :

$$
\bar{V}=V_{\mathrm{M}}+\Delta V_{\mathrm{s}} .
$$

The molecular volume consists of the van der Waals volume $V_{\mathrm{W}}$, which is the sum of the van der Waals volumes of all the solute constitutive atoms, and the void volume $V_{\mathrm{V}}$, which is the volume of structural voids within the solvent-inaccessible core of the solute that result from imperfect atomic packing. The solvation volume also includes two contributions. One is the so-called thermal volume $V_{\mathrm{T}}$ which results from thermally induced molecular fluctuation between the solute and solvent and is considered as average empty space around the solute due to imperfect packing of solvent. The other is the change in the solvent volume induced by the intermolecular interaction between the solute and solvent, which is referred to as the interaction volume $V_{\mathrm{I}}$. There is another contribution, though it is not explicitly given in the above equation. It is the ideal contribution only from the translational degrees of freedom of the solute, which is given by $k_{\mathrm{B}} T \chi_{\mathrm{T}}^{0}$ irrespective of the chemical properties of the solute. Thus, the volume decomposition is reexpressed by

$$
\bar{V}=V_{\mathrm{W}}+V_{\mathrm{V}}+V_{\mathrm{T}}+V_{\mathrm{I}}+k_{\mathrm{B}} T \chi_{\mathrm{T}}^{0} .
$$

All the volume terms can be defined and calculated within the framework of the 3D-RISM-KB theory in combination with the conventional geometric volume calculation [64,67]. The van der Waals $V_{\mathrm{W}}$ is the volume occupied by the atomic spheres. The void volume $V_{\mathrm{V}}$ is defined as void space inside the solute and its surface that the solvent probe cannot access. The two geometric terms are obtained from the conventional volume calculation [11-13], using the atomic diameters converted from the LJ parameters employed in the 3D-RISM calculation. In a possible conversion, the diameter of each atom is set to the distance where the LJ potential energy reaches the thermal energy $k_{\mathrm{B}} T$. This definition has been found to be reasonable [67]. The thermal volume $V_{\mathrm{T}}$ is defined by $\bar{V}_{0}-k_{\mathrm{B}} T \chi_{\mathrm{T}}^{0}-\left(V_{\mathrm{W}}+V_{\mathrm{V}}\right)$, where $\bar{V}_{0}$ is the PMV of a hypothetical molecule whose atomic charges are completely removed. The interaction volume $V_{\mathrm{I}}=\bar{V}-\bar{V}_{0}$ is defined as the contribution of the 
electrostatic interaction. The PMVs $\bar{V}$ and $\bar{V}_{0}$ are calculated from the 3D-RISM-KB theory. The ideal term $k_{\mathrm{B}} T \chi_{\mathrm{T}}^{0}$ is naturally included in the $3 \mathrm{D}-\mathrm{KB}$ equation (23).

We have applied the volume decomposition to the PMV of several peptides and proteins [67]. The results are shown in figure 3. The ideal term is omitted from the figure because it takes a small constant value of $1 \mathrm{~cm}^{3} \mathrm{~mol}^{-1}$ under the thermodynamic condition of ambient water. It is obvious that the van der Waals volume accounts for the most part of the PMV. The void volume also makes up a significant part of the PMV. In contrast, the solvation components, especially the interaction volume, make only a minor contribution to the PMV. However, this result never implies that the solvation components are insignificant in analyzing the PMV of proteins. When we consider the volume change upon a protein reaction such as a conformational transition, it is most likely that the change in the van der Waals volume is negligible unless unusual van der Waals overlaps occur. In such cases, the solvation effects would become significant. In fact, the thermal volume always plays an important role in the PMV changes upon protein reactions. Two instances of the kind will be presented in the following subsections. In addition, further careful analysis showed that there is a nearly linear relation between the thermal volume and the protein surface area, which is known as the solvent-accessible surface area (ASA). The interaction volume is also roughly proportional to the ASA. However, there is a complicated correlation with chemical properties of the functional groups exposed to aqueous phase.

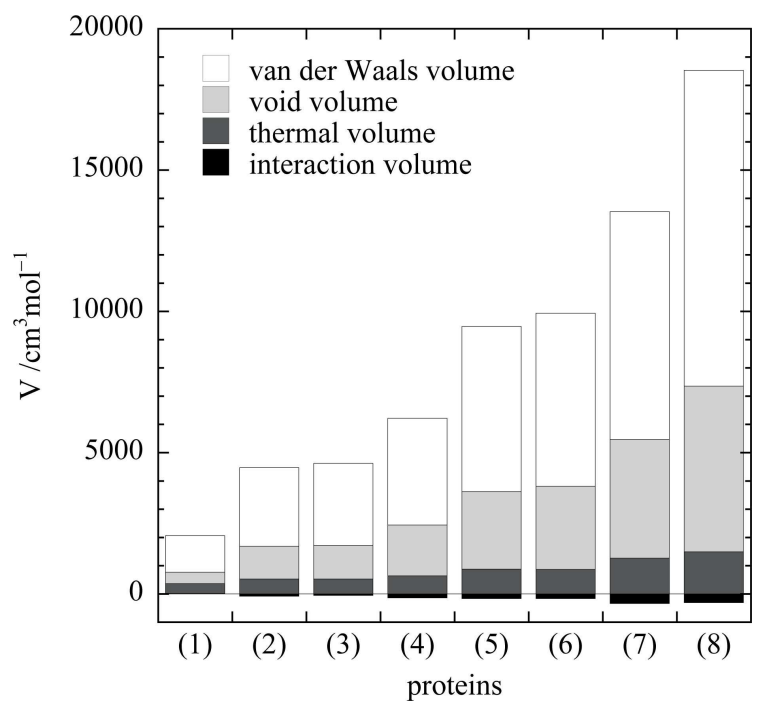

Figure 3. Volume components in the PMV of eight representative peptides and proteins, (1) honey bee venom melittin, (2) sea snake venom erabutoxin B, (3) bovine pancreatic trypsin inhibitor (BPTI), (4) human erythrocyte ubiquitin, (5) bovine pancreatic ribonuclease (RNase) A, (6) hen egg-white lysozyme, (7) bovine milk $\beta$-lactoglobulin A, and (8) bovine pancreatic $\alpha$-chymotrypsinogen A, calculated by the 3D-RISM/HNC theory in combination with the geometric calculation.

It should be noted here that if we employ the conventional RISM theory instead of the 3DRISM theory, this volume decomposition is not effective in analyzing the PMV. That is because the RISM theory cannot completely incorporate the contribution of the molecular volume into the $\mathrm{PMV}$, as described above. Even in that case, the estimation of the interaction volume $V_{\mathrm{I}}=\bar{V}-\bar{V}_{0}$ is valid, because the molecular volume contributions included in $\bar{V}$ and $\bar{V}_{0}$ will be cancelled.

Before closing this subsection, it is worth briefly explaining the relations to other volume contributions generally used. The intrinsic volume defined in the SPT [20] conceptually corresponds to $V_{\mathrm{W}}+V_{\mathrm{V}}+V_{\mathrm{T}}$. The solvent-excluded volume, which is defined as the volume of the space where any center of solvent molecules cannot access [11], contains the molecular volume and the additional volume on the surface. However, the latter contribution cannot be related directly to any solvent contribution in this volume decomposition. 


\subsection{Volume change upon structural transition of protein}

The pressure-induced structural transition of proteins has been continuously attracting the attention of biochemists and biophysicists [92-94]. As described in Introduction, the molecular mechanism can be revealed by analyzing the PMV change upon the transition through equation (3). We have recently applied the 3D-RISM theory to the investigation of the PMV change of a protein, ubiquitin, associated with a conformational transition induced by pressure [71]. Ubiquitin is one of the few proteins whose 3D structural data in aqueous solution under high pressure have been resolved. The low- and high-pressure structures (LPS and HPS) under 3 MPa and 300 MPa, respectively, were determined by high-pressure NMR technique [95]. The LPS and HPS are shown in figure 4.

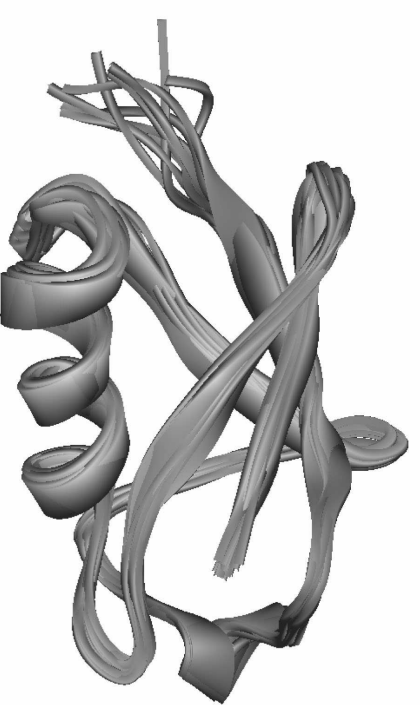

low-pressure structures

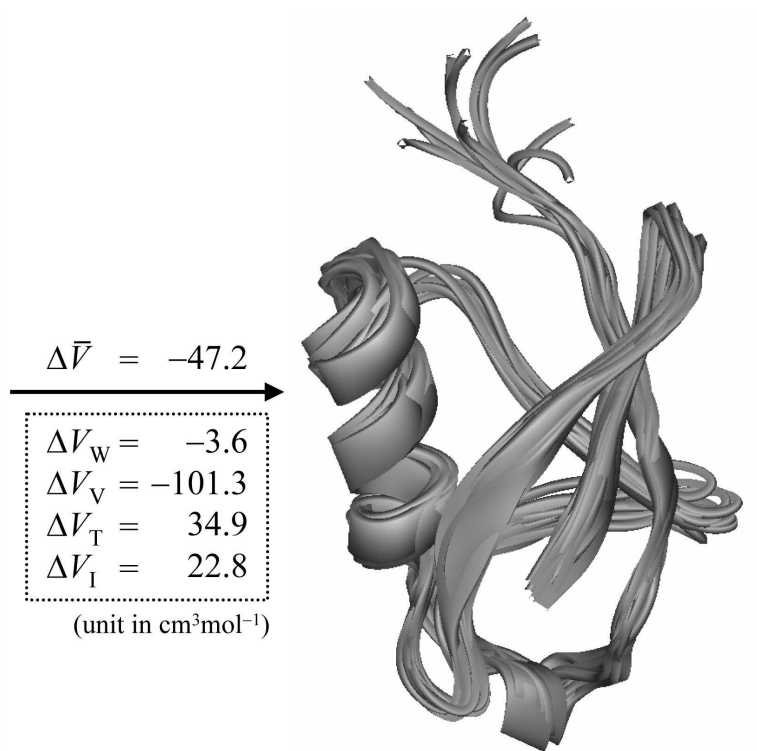

high-pressure structures

Figure 4. Solid ribbon representation of low-pressure $(3 \mathrm{MPa})$ and high-pressure (300 $\mathrm{MPa})$ structures of ubiquitin. Each ten superimposed structures included in PDB files 1V80 and 1V81 are displayed. The theoretical values of the partial molar volume change and its components are also given.

The PMV values of the LPS and HPS calculated by the 3D-RISM/HNC theory were $5788.4 \mathrm{~cm}^{3} \mathrm{~mol}^{-1}$ and $5741.2 \mathrm{~cm}^{3} \mathrm{~mol}^{-1}$, respectively, each of which is the average value for the ten most probable structures from NMR data. The PMV decreases by $-47.2 \mathrm{~cm}^{3} \mathrm{~mol}^{-1}$ accompanying the pressure-induced structural transition. The theoretical result is in fair agreement with the corresponding experimental data, $-24 \mathrm{~cm}^{3} \mathrm{~mol}^{-1}[96]$. Then, we decomposed the PMV change into the volume components described above. The changes in the van der Waals, void, thermal, and interaction volumes were $\Delta V_{\mathrm{W}}=-3.6 \mathrm{~cm}^{3} \mathrm{~mol}^{-1}, \Delta V_{\mathrm{V}}=-101.3 \mathrm{~cm}^{3} \mathrm{~mol}^{-1}, \Delta V_{\mathrm{T}}=34.9 \mathrm{~cm}^{3} \mathrm{~mol}^{-1}$, and $\Delta V_{\mathrm{I}}=22.8 \mathrm{~cm}^{3} \mathrm{~mol}^{-1}$, respectively. The result demonstrates that the total PMV reduction is primarily caused by the decrease in the void volume, which is partially cancelled by the increases in the two hydration contributions. The van der Waals volume change is negligible, as the pressure did not cause any unusual van der Waals overlaps. The volume changes can be related to the changes in the protein structure as follows. The decrease in the void volume indicates a partial loss of the structural voids in the protein. The increase in the thermal volume implies the generation of additional empty space around the protein, primarily due to the extension of the protein surface.

Based on those findings, we concluded that the PMV reduction was caused by the penetration of water molecules into the protein interior. That is because the water penetration can eliminate the void space within the protein and at the same time can expand the protein surface. It is an important point that the former rather than the latter effect governs the total PMV change. 
Further detailed analysis, in which the contributions of the volume changes from some fragments of the protein were estimated, showed that the total volume changes were primarily determined by the volume changes of a certain small part of the protein. It follows that the water molecules penetrated into the particular part of the protein. Actually, the water penetration was confirmed in terms of the water distribution around the protein, which was also obtained by the 3D-RISM theory.

In fact, penetration of water into a protein is considered to be a potential candidate for the driving force of the pressure-induced denaturation (unfolding) of proteins [92,93]. The theoretical analysis of volume decomposition proved that the water penetration reduces the PMV of a protein. This implies that the increasing pressure stabilizes the structure swelled by the water penetration, which would be the denatured structure itself or its precursor.

\subsection{Volume change upon ligand binding to protein}

The volume decomposition based on the 3D-RISM theory has also been applied to the analysis of the PMV change accompanying a ligand-protein association, xenon-lysozyme binding [68]. Actually, that study was performed for elucidating the molecular mechanisms of the pressure reversal of general anesthesia. The pressure reversal phenomenon is an important feature of general anesthesia [97-100]. Currently, it is believed that anesthetics interact with specific sites of transmembrane proteins such as ion channels, which regulate signal transduction [101,102]. It is considered that the anesthetic-protein binding is a physicochemical or thermodynamic process at the molecular level. In that case, the pressure reversal of anesthesia should also be explained by the thermodynamic relation (3); in other words, the question "why and how the anesthetic-protein binding expands the PMV" should be answered. We chose the xenon-lysozyme binding as a model system because the xenon binding sites in lysozyme had been resolved by X-ray analysis [103] and xenon is an actual anesthetic.

Lysozyme has two xenon binding sites: one corresponds to the binding pocket of native ligands (substrate binding site), the other is located in a cavity inside the protein (internal site) [103], which are shown in figure 5. Using the 3D structures of the two xenon-lysozyme complexes modelled

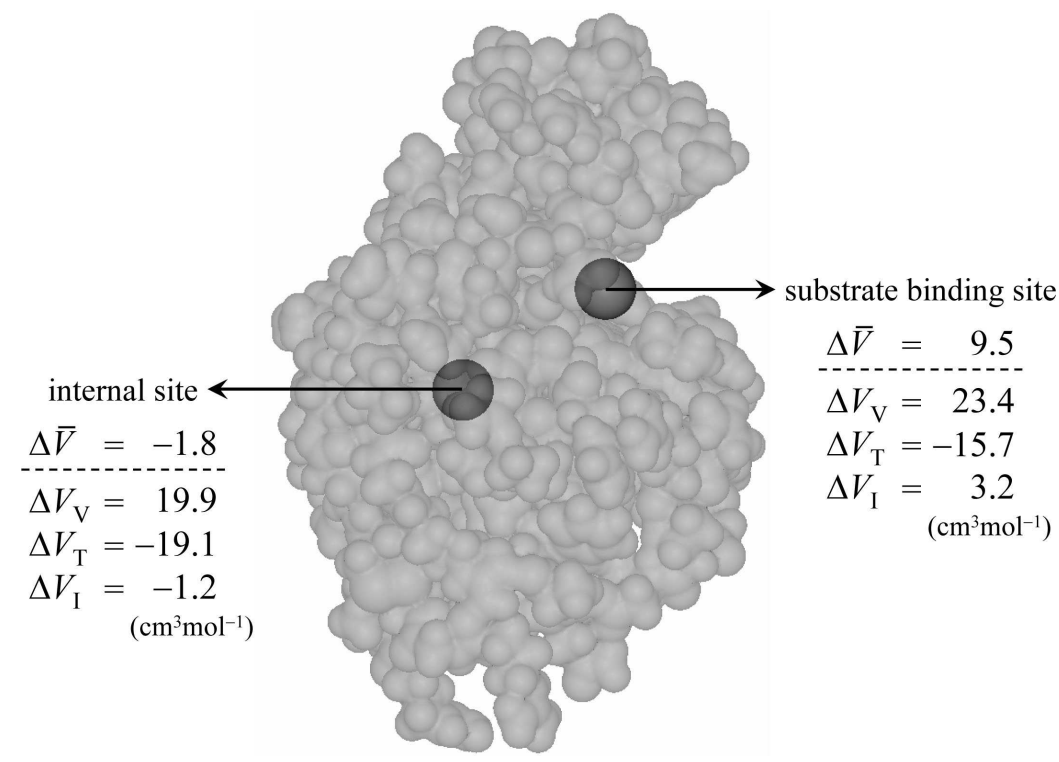

Figure 5. Van der Waals surface representation of the structure of xenon-lysozyme complex. The theoretical values of the partial molar volume changes and its components accompanying the xenon binding to the two sites are also presented.

on the basis of the NMR structure of lysozyme in aqueous solution and the X-ray structure of xenon-lysozyme complex [104], we calculated the PMV changes associated with the xenon binding 
to the two binding sites by the 3D-RISM/HNC theory. The PMV changes were $9.5 \mathrm{~cm}^{3} \mathrm{~mol}^{-1}$ at the substrate binding site and $-1.8 \mathrm{~cm}^{3} \mathrm{~mol}^{-1}$ at the internal site. On the basis of equation (3), the result implies that the increasing pressure reduces the binding constant of xenon to the substrate binding site, whereas it has little effect on the binding to the internal site. The former is in accordance with the pressure reversal phenomenon of anesthesia. To find the factors causing the PMV changes, we carried out the volume decomposition analysis. The result showed that $\Delta V_{\mathrm{V}}=23.4 \mathrm{~cm}^{3} \mathrm{~mol}^{-1}, \Delta V_{\mathrm{T}}=-15.7 \mathrm{~cm}^{3} \mathrm{~mol}^{-1}$, and $\Delta V_{\mathrm{I}}=3.2 \mathrm{~cm}^{3} \mathrm{~mol}^{-1}$ for the substrate binding site, and $\Delta V_{\mathrm{V}}=19.9 \mathrm{~cm}^{3} \mathrm{~mol}^{-1}, \Delta V_{\mathrm{T}}=-19.1 \mathrm{~cm}^{3} \mathrm{~mol}^{-1}$, and $\Delta V_{\mathrm{I}}=-1.2 \mathrm{~cm}^{3} \mathrm{~mol}^{-1}$ for the internal site. For the both cases, the changes in $V_{\mathrm{W}}$ were negligible, and the changes in the ideal term were $-1.3 \mathrm{~cm}^{3} \mathrm{~mol}^{-1}$, which is only the ideal effect due to the association of two molecules.

The volume changes are characterized by the compensation between the void volume changes and the thermal volume changes. This is explained as follows. Prior to the binding, xenon as well as the binding sites are hydrated and have some empty space between them and water molecules, which is involved in the thermal volume. When xenon binds to the sites, they are dehydrated and the corresponding thermal volume vanishes; at the same time, some void space between xenon and the binding sites is created. The cancellation was incomplete and the void volume expansion dominated at the substrate binding site, whereas the cancellation was almost complete at the internal site. The result was supported by the changes in the coordination numbers of water molecules at the sites, which were calculated from the distribution functions of water that were also obtained from the 3D-RISM theory. In fact, accompanying the xenon binding, one xenon atom expelled two water molecules from the substrate binding site, whereas it expelled one water molecule from the internal site. The former result implies that the packing between xenon and the substrate binding site is looser than that between water and the site. The loose binding is most likely to make additional void space. In the latter case, xenon fits into the internal site as water does.

It is interesting that the interaction volume did not significantly contribute to the PMV changes. In fact, some early workers proposed that the PMV expansion upon the anesthetic-protein binding is mainly due to the release of "freezing" or "electrostricted" water molecules on the protein surface $[98,99]$. The effect should be included in the interaction volume change in our volume decomposition scheme. However, our results demonstrated that the interaction volume changes are not significant to the PMV change associated with the xenon-lysozyme binding. Our model study argues that the packing between ligand and protein is of primary importance for considering the PMV change. The pressure reversal of anesthesia can be caused by loose packing between the anesthetics and the action site. The phenomenon can be distinguished from tight binding with ordinary ligands which would not undergo a significant volume change.

\subsection{Summary}

It has been demonstrated that the 3D-RISM theory can reproduce the experimental data for the PMV of proteins and its changes as well. Moreover, the volume decomposition analysis provides a molecular picture of the PMV changes of protein reactions. A change in the PMV is determined not only by changes in the geometry but also by changes in the solvation. In most cases, the balance between the changes in the geometric and solvation volumes, especially in the void and thermal volumes, is the most significant to the total PMV change. In other words, the difference between the intramolecular packing of solute (protein) and the solute-solvent intermolecular packing can cause the PMV change. The solute-solvent electrostatic interaction can also contribute to the PMV change, depending on the reaction process. However, the interaction volume has a complicated relation to the protein structure, which has not been fully comprehended. 


\section{Effects of intramolecular fluctuation on the partial molar volume}

\subsection{Equilibrium partial molar volume in the conformational ensemble}

So far we have calculated the PMV of a solute molecule by assuming that the molecule is rigid and fixed to a single conformation. However, solute molecules should fluctuate in the conformational space in actual solution. In this section, we consider the effects of the intramolecular fluctuation on the PMV.

Starting with the equilibrium chemical potential of a solute molecule at infinite dilution, we derived the equilibrium PMV [53]. It is given by

$$
\bar{V}_{\mathrm{eq}}=\langle\bar{V}(\Phi)\rangle
$$

where the bracket denotes the conformational ensemble average of a function depending on the internal coordinate $\Phi$. For example, the conformational ensemble average of a function $f(\Phi)$ is defined by

$$
\langle f(\Phi)\rangle \equiv \frac{\int f(\Phi) \exp \left(-\beta\left(U(\Phi)+\mu^{\operatorname{ex}}(\Phi)\right)\right) \mathrm{d} \Phi}{\int \exp \left(-\beta\left(U(\Phi)+\mu^{\operatorname{ex}}(\Phi)\right)\right) \mathrm{d} \Phi},
$$

where $U(\Phi)$ is the conformational energy in real gas phase and $\mu^{\mathrm{ex}}(\Phi)$ is the excess chemical potential or the solvation free energy. As a result, the equilibrium PMV is expressed simply by the conformational average of the PMVs of conformers, though the result is actually not trivial.

We can calculate the equilibrium PMV by combining the (3D-)RISM theory with an appropriate molecular simulation [105-107]. In the hybrid method, various conformers are sampled by the simulation and the PMV of each conformer is calculated by the (3D-)RISM theory. The solvation free energy of each conformer, which is necessary in the calculation of equation (27), can be also obtained from the (3D-)RISM theory [40,108].

The intramolecular fluctuation has only a minor effect on the total quantity of the PMV of a protein. However, our recent study [65] suggests that the intramolecular fluctuation is significant when we consider the PMV change of a protein reaction, because the PMV fluctuation is comparable in magnitude to the PMV change.

\subsection{Equilibrium partial molar compressibility in the conformational ensemble}

The equilibrium partial molar compressibility (PMC) is generally defined by the pressure derivative of the equilibrium PMV:

$$
\bar{K}_{\mathrm{eq}} \equiv-\left(\frac{\partial \bar{V}_{\mathrm{eq}}}{\partial p}\right)_{\mathrm{T}},
$$

where we consider the isothermal compressibility rather than the adiabatic one. (PMC takes slightly different definitions such as $-\left(1 / \bar{V}_{\text {eq }}\right)\left(\partial \bar{V}_{\text {eq }} / \partial p\right)$, depending on the field where it is used.) The intramolecular fluctuation has a more essential effect on the PMC than on the PMV. If we assume that a protein alone can be regarded as a thermodynamic system of the NPT ensemble and that the coupling between the protein and the solvent is negligible, the protein compressibility $K_{\mathrm{p}}$ is related to the mean square fluctuation of the protein volume $V_{\mathrm{p}}$ [109]:

$$
K_{\mathrm{p}}=\beta\left\langle\left(\delta V_{\mathrm{p}}\right)^{2}\right\rangle
$$

where $\delta V_{\mathrm{p}}=V_{\mathrm{p}}-\left\langle V_{\mathrm{p}}\right\rangle$. Under this assumption, the compressibility of a protein with a fixed conformation is zero. Thus, the protein fluctuation is necessary for considering the protein compressibility. However, the assumption is probably not realized in a real solution system. In what follows, we consider the actual intramolecular fluctuation effect on the PMC, which should be coupling with the solvation effect.

From equations (26) and (28), we finally obtained the statistical-mechanical expression of the PMC [53]:

$$
\bar{K}_{\mathrm{eq}}=\langle\bar{K}(\Phi)\rangle+\beta\left\langle(\delta \bar{V}(\Phi))^{2}\right\rangle,
$$


where $\bar{K}(\Phi)=-(\partial \bar{V}(\Phi) / \partial p)$ is the PMC of the conformer fixed to an internal coordinate $\Phi$. The first term results from perturbation of the solvation structure by the pressure, which causes a change in the PMV of each fixed conformer. The second term is the mean square fluctuation of the PMV, which also includes the solvation effect, unlike the protein volume in equation (29).

Just like the PMV (equation (24)), the PMC can be decomposed into the molecular (or intrinsic) $K_{\mathrm{M}}$ and the solvation $\Delta K_{\mathrm{s}}$ terms $[8,110,111]$ :

$$
\bar{K}_{\mathrm{eq}}=K_{\mathrm{M}}+\Delta K_{\mathrm{s}} .
$$

By substituting equation (24) into equation (30), we obtain explicit expressions of the two terms using the molecular volume $V_{\mathrm{M}}$ and the solvation volume $\Delta V_{\mathrm{s}}$ :

$$
\begin{aligned}
K_{\mathrm{M}} & =\beta\left\langle\left(\delta V_{\mathrm{M}}(\Phi)\right)^{2}\right\rangle, \\
\Delta K_{\mathrm{s}} & =\beta\left\langle\left(\delta \Delta V_{\mathrm{s}}(\Phi)\right)^{2}\right\rangle+2 \beta\left\langle\delta V_{\mathrm{M}}(\Phi) \delta \Delta V_{\mathrm{s}}(\Phi)\right\rangle-\left\langle\frac{\partial \Delta V_{\mathrm{s}}(\Phi)}{\partial p}\right\rangle,
\end{aligned}
$$

where the reasonable assumption that the molecular volume does not depend on the pressure is adopted. The molecular compressibility is the mean square fluctuation of the molecular volume, which corresponds to equation (29). This is contributed exclusively due to the fluctuation of the void volume, since the van der Waals volume takes an almost constant value regardless of the conformation. The solvation compressibility consists of three terms. The first term is the contribution of the mean square fluctuation of the solvation volume. The second one is not a pure contribution from the solvation, but is the coupling term of the molecular volume fluctuation and the solvation volume fluctuation. The last one is the effect of the solvation structure change by the pressure. (It implicitly includes the ideal contribution: $-k_{\mathrm{B}} T\left(\partial \chi_{\mathrm{T}}^{0} / \partial p\right)$.)

The experimentally observed values of the equilibrium PMC of proteins, which were converted from adiabatic to isothermal [8], are commonly positive. The molecular compressibility is necessarily positive by its definition. On the other hand, the solvation compressibility is generally considered to be negative from empirical viewpoint $[110,111]$. Since the first term should be positive, the negative contribution comes from the second or third term or both. The negative correlation between the void volume and the thermal volume [65] implies a negative contribution of the second term. The third term can also contribute negatively. In fact, the PMC values for small polar organic compounds such as amino acids, for which it can be assumed that the fluctuation effects are negligible, are negative $[112,113]$.

The molecular compressibility of proteins has been investigated by molecular simulation [114122]. (In some of those studies, other geometric volumes such as the solvent-excluded volume and the Voronoi volume were used instead of the molecular volume.) However, a complete evaluation of the solvation compressibility has not been carried out so far. The hybrid approach of the (3D)RISM theory and molecular simulation described above enables us to calculate the equilibrium PMC and to determine the molecular and solvation compressibility terms. Work in this direction is in progress.

\section{Concluding remarks}

The PMV is a thermodynamic rather than a geometric quantity. Although the geometric volume contributions are dominant in the total quantity of the PMV of large molecules such as proteins, the solvation has substantial effects on the PMV changes associated with chemical reactions, including structural transition of protein and ligand-protein binding. When we employ a theory in order to analyze a PMV change, the theory should be capable of describing the solvation effects. As introduced above, the RISM theory, especially its 3D generalization version, is currently the most promising method of investigating the PMV for various molecular systems, including biomolecular systems. In the theory, the solvation effects are naturally included in the PMV because it is directly calculated from the correlation functions between the solute and solvent, or the distribution of solvent around the solute, obtained by the theory. It is noteworthy that the 3D-RISM theory 
is capable of reproducing the water molecules isolated and confined in the protein interior as well as the ordinary hydration $[123,124]$. The finding means that the theory successfully takes into consideration the effects from each individual solvent molecule into the PMV. Thus, the 3DRISM theory has a great advantage over other conventional methods in investigating the PMV of complicated biomolecules.

\section{Acknowledgement}

The author would like to express his appreciation to all of his collaborators, especially to Profs. F. Hirata, M. Kinoshita, and A. Kovalenko for their continuing guidance and encouragement.

\section{References}

1. Millero F.J., Chem. Rev., 1971, 71, 147.

2. Planck M., Ann. Phys., 1887, 32, 462.

3. Evans M.G., Polanyi M., Trans. Faraday Soc., 1935, 31, 875.

4. Asano T., le Noble W.J., Chem. Rev., 1978, 78, 407.

5. Høiland H., Thermodynamic Data for Biochemistry and Biotechnology. edited by Hing H.-J., Springer-Verlag, Verlin, 1986, p. 17.

6. Zamyatnin A.A., Ann. Rev. Biophys. Bioeng., 1984, 13, 145.

7. Zhao H., Biophys. Chem., 2006, 122, 157.

8. Gekko K., Hasegawa Y., Biochemistry, 1986, 25, 6563.

9. Royer C.A., Biochim. Biophys. Acta, 2002, 1595, 201.

10. Bondi A., J. Phys. Chem., 1964, 68, 441.

11. Richards F.M., Ann. Rev. Biophys. Bioeng., 1977, 6, 151.

12. Connolly M.L., J. Am. Chem. Soc., 1985, 107, 1118.

13. Liang J., Edelsbrunner H., Fu P., Sudhakar P.V., Subramaniam S., Proteins: Struct. Funct. Genet., 1998, 33, 1.

14. Cabani S., Gianni P., Mollica V., Lepori L., J. Solution Chem., 1981, 10, 563.

15. Kharakoz D.P., J. Solution Chem., 1992, 21, 569.

16. Traube J., Samml. Chem. Chemischtech. Vorträge, 1899, 4, 255.

17. Reiss H., Frisch H.L., Lebowitz J.L., J. Chem. Phys., 1959, 31, 369.

18. Reiss H., Adv. Chem. Phys., 1965, 9, 1.

19. Pierotti R.A., Chem. Rev., 1976, 76, 717.

20. Hirata F., Arakawa K., Bull. Chem. Soc. Jpn., 1973, 46, 3367.

21. Pierotti R.A., J. Phys. Chem., 1963, 67, 1840.

22. Pierotti R.A., J. Phys. Chem., 1965, 69, 281.

23. Klapper M.H., Biochim. Biophys. Acta, 1971, 229, 557.

24. Goldman S., J. Phys. Chem., 1977, 81, 1428.

25. Matteoli E., Z. Phys. Chem., 1980, 123, 141.

26. Lee B., J. Phys. Chem., 1983, 87, 112.

27. Graziano G., J. Chem. Phys., 2006, 124, 134507.

28. Irisa M., Nagayama K., Hirata F., Chem. Phys. Lett., 1993, 207, 430.

29. Hirata F., Imai T., Irisa M., Rev. High Pressure Sci. Technol., 1998, 8, 96.

30. Irisa M., Takahashi T., Nagayama K., Hirata F., Mol. Phys., 1995, 85, 1227.

31. Yoshimura Y., Nakahara M., J. Chem. Phys., 1984, 81, 4080.

32. Ben-Amotz D., J. Phys. Chem., 1993, 97, 2314.

33. Kirkwood G., Buff F.P., J. Chem. Phys., 1951, 19, 774.

34. Pratt L.R., Pohorille A., Proceedings of the EBSA International Workshop on Water-Biomolecule Interactions. edited by Palma M.U., Palma-Vittorelli M.B., Parak F., Societá Italiana de Fisica, Bologna, 1993, p. 261.

35. Paulaitis M.E., Ashbaugh H.S., Garde S., Biophys. Chem., 1994, 51, 349.

36. Matubayasi N., Levy R.M., J. Phys. Chem., 1995, 100, 2681.

37. Lockwood D.M., Rossky P.J., J. Phys. Chem. B, 1999, 103, 1982.

38. Lockwood D.M., Rossky P.J., Levy R.M., J. Phys. Chem. B, 2000, 104, 4210.

39. Hansen J.-P., McDonald I.R., Theory of Simple Liquids, 3rd ed., Academic Press, London, 2006.

40. Hirata F. (ed.), Molecular Theory of Solvation, Kluwer, Dordrecht, 2003. 
41. Fries P.H., Patey G.N., J. Chem. Phys., 1985, 82, 429.

42. Chandler D., Andersen H.C., J. Chem. Phys., 1972, 57, 1930.

43. Chandler D., J. Chem. Phys., 1973, 59, 2749.

44. Kusalik P.G., Patey G.N., J. Chem. Phys., 1988, 88, 7715.

45. Kusalik P.G., Patey G.N., J. Chem. Phys., 1988, 89, 5843.

46. Hirata F., Rossky P.J., Chem. Phys. Lett., 1981, 83, 329.

47. Hirata F., Pettitt B.M., Rossky P.J., J. Chem. Phys., 1982, 77, 509.

48. Hirata F., Rossky P.J., Pettitt B.M., J. Chem. Phys., 1983, 78, 4133.

49. Hirata F., Bull. Chem. Soc. Jpn., 1998, 71, 1483.

50. Chong S.-H., Hirata F., J. Phys. Chem. B, 1997, 101, 3209.

51. Imai T., Nomura H., Kinoshita M., Hirata F., J. Phys. Chem. B, 2002, 106, 7308.

52. Leu L., Blankschtein D., J. Phys. Chem., 1992, 96, 8582.

53. Imai T., Hirata F., J. Chem. Phys., 2003, 119, 5623.

54. Imai T., Hirata F., J. Chem. Phys., 2005, 122, 094509.

55. Imai T., Kinoshita M., Hirata F., J. Chem. Phys., 2000, 112, 9469.

56. Kinoshita M., Imai T., Kovalenko A., Hirata F., Chem. Phys. Lett., 2001, 348, 337.

57. Takekiyo T., Imai T., Kato M., Taniguchi Y., Biopolymers, 2004, 73, 283.

58. Ohba M., Kawaizumi F., Nomura H., J. Phys. Chem., 1992, 96, 5129.

59. Amakasu Y., Ohba M., Kawaizumi F., Nomura H., J. Phys. Chem., 1995, 99, 9258.

60. Koga K., Tanaka H., Zeng X.C., J. Phys. Chem., 1996, 100, 16711.

61. Beglov D., Roux B, J. Phys. Chem. B, 1997, 101, 7821.

62. Kovalenko A., Hirata F., Phys. Chem. Lett., 1998, 290, 237.

63. Harano Y., Imai T., Kovalenko A., Kinoshita M., Hirata F., J. Chem. Phys., 2001, 114, 9506.

64. Imai T., Harano Y., Kovalenko A., Hirata F., Biopolymers, 2001, 59, 512.

65. Imai T., Takekiyo T., Kovalenko A., Hirata F., Kato M., Taniguchi Y., Biopolymers, 2005, 79, 97.

66. Imai T., Kovalenko A., Hirata F., Chem. Phys. Lett., 2004, 395, 1.

67. Imai T., Kovalenko A., Hirata F., J. Phys. Chem. B, 2005, 109, 6658.

68. Imai T., Isogai H., Seto T., Kovalenko A., Hirata F., J. Phys. Chem. B, 2005, 110, 12149.

69. Imai T., Kovalenko A., Hirata F., Mol. Simul., 2006, 32, 817.

70. Yamazaki T., Imai T., Hirata F., Kovalenko A., J. Phys. Chem. B, 2007, 111, 1206.

71. Imai T., Ohyama S., Kovalenko A., Hirata F. Protein Sci., in press.

72. Morita T., Hiroike K., Prog. Theor. Phys., 1960, 23, 1003.

73. Perkyns J.S., Pettitt B.M., Chem. Phys. Lett., 1992, 190, 626.

74. Perkyns J., Pettitt B.M., J. Chem. Phys., 1992, 97, 7656.

75. Wang J., Cieplak P., Kollman P.A., J. Comput. Chem., 2000, 21, 1049.

76. Berendsen H.J.C., Grigera J.R., Straatsma T.P., J. Phys. Chem., 1987, 91, 6269.

77. Kovalenko A., Hirata F., J. Chem. Phys., 1999, 110, 10095.

78. Imai T. (unpublished).

79. Verlet L., Mol. Phys., 1980, 41, 183.

80. Kovalenko A., Hirata F., J. Chem. Phys., 2000, 113, 2793.

81. Weeks J.D., Chandler D., Andersen J., J. Chem. Phys., 1971, 54, 5237.

82. Martynov G.A., Sarkisov G.N., Mol. Phys., 1984, 49, 1495.

83. Ballone P., Pastore G., Galli G., Gazzillo D., Mol. Phys., 1986, 59, 275.

84. Zana R., Yeager E., J. Phys. Chem., 1967, 71, 521.

85. Yamaguchi T., Matsuoka T., Koda S., J. Chem. Phys., 2003, 119, 4437.

86. Kovalenko A., Hirata F., J. Chem. Phys., 2000, 112, 10391.

87. Harano Y. (private communication).

88. Tikhonov D.A. (data from the Web site, URL: http://rismproteins.org/).

89. Chalikian T.V., Ann. Rev. Biophys. Biochem. Struct., 2003, 32, 207.

90. Kauzmann W., Adv. Protein Chem., 1959, 14, 1.

91. Chalikian T.V., Breslauer K.J., Biopolymers, 1996, 39, 619.

92. Balny C., Masson P., Heremans K., Biochim. Biophys. Acta, 2002, 1595, 3.

93. Meersman F., Dobson C.M., Heremans K., Chem. Soc. Rev., 2006, 35, 908.

94. Akasaka K., Chem. Rev., 2006, 106, 1814.

95. Kitahara R., Yokoyama S., Akasaka K., J. Mol. Biol., 2005, 347, 277.

96. Kitahara R., Yamada H., Akasaka K., Biochemistry, 2001, 40, 13556.

97. Miller K.W., Paton W.D.M., Smith R.A., Smith E.B., Mol. Pharmacol., 1973, 9, 131.

98. Eyring H., Woodbury J.W., D’Arrigo J.A., Anesthesiology, 1973, 38, 415. 
99. Ueda I., Mashimo T., Physiol. Chem. Phys., 1982, 14, 157.

100. Wlodarczyk A., McMillan P.F., Greenfield S.A., Chem. Soc. Rev., 2006, 35, 890.

101. Franks N.P., Lieb W.R., Nature, 1994, 367, 607.

102. Miller K.W., Br. J. Anaesth., 2002, 89, 17.

103. Prangé T., Schiltz M., Pernot L., Colloc'h N., Longhi S., Bourguet W., Fourme R., Proteins: Struct. Funct. Genet., 1998, 30, 61.

104. Isogai H., Seto T., Nasaka S., Int. Congr. Ser., 2005, 1283, 318.

105. Kinoshita M., Okamoto Y., Hirata F., J. Am. Chem. Soc., 1998, 120, 1855.

106. Kinoshita M., Okamoto Y., Hirata F., J. Chem. Phys., 1999, 110, 4090.

107. Mitsutake A., Kinoshita M., Okamoto Y., Hirata F., Chem. Phys. Lett., 2000, 329, 295.

108. Singer S.J., Chandler D., Mol. Phys., 1985, 55, 621.

109. Cooper A., Proc. Natl. Acad. Sci. USA, 1976, 73, 2740.

110. Kharakoz D.P., Sarvazyan A.P., Biopolymers, 1993, 33, 11.

111. Chalikian T.V., Sarvazyan A.P., Breslauer K.J., Biophys. Chem., 1994, 51, 89.

112. Millero F.J., Surdo A.L., Shin C., J. Phys. Chem., 1978, 82, 784.

113. Kharakoz D.P., J. Phys. Chem., 1991, 95, 5634.

114. Kitchen D.B., Reed L.H., Levy R.M., Biochemistry, 1992, 31, 10083.

115. Yamato T., Higo J., Seno Y., Go N., Proteins: Struct. Funct. Genet., 1993, 16, 327.

116. Gerstein M., Tsai J., Levitt M., J. Mol. Biol., 1995, 249, 955.

117. Paci E., Marchi M., Proc. Natl. Acad. Sci. USA, 1996, 93, 11609.

118. Kobayashi N., Yamato T., Go N., Proteins: Struct. Funct. Genet., 1997, 28, 109.

119. Tama F., Miyashita O., Kitao A., Go N., Eur. Biophys. J., 2000, 29, 472.

120. Dadarlat V.M., Post C.B., J. Phys. Chem. B, 2001, 105, 715.

121. Marchi M., J. Phys. Chem. B, 2003, 107, 6598.

122. Mori K., Seki Y., Yamada Y., Matsumoto H., Soda K., J. Chem. Phys., 2006, 125, 054903.

123. Imai T., Hiraoka R., Kovalenko A., Hirata F., J. Am. Chem. Soc., 2005, 127, 15334.

124. Imai T., Hiraoka R., Kovalenko A., Hirata F., Proteins: Struct. Funct. Bioinformat., 2007, 66, 804.

\section{Молекулярна теорія парціального молярного об'єму і її застосування до біомолекулярних систем}

\section{T.Imai}

Факультет біології та біоінформатики, університет Ріцумейкан, Кусацу, Японія

Отримано 3 травня 2007, в остаточному вигляді - 8 червня 2007

Парціальний молярний об'єм (PMV) є термодинамічною величиною, яка містить важливу інформацію про взаємодії розчинена речовина-розчинник, а також про структуру розчиненої речовини в розчині. Крім того, PMV є найбільш суттєвою величиною при аналізі впливу тиску на перебіг хімічної реакції. Ця стаття містить огляд сучасного стану молекулярних теорій PMV, зокрема, молекулярної теорії базисної моделі взаємодіючих центрів (RISM) і їі тривимірного узагальнення (3D-RISM), які, для обчислення PMV, є об’єднані з теорією Кірквуда-Баффа для розчинів. Тут також представлені застосування теорії для ряду випадків, що стосуються PMV біомолекул. Ми також коротко обговорюємо вплив міжмолекулярних флуктуацій на PMV, який є важливими для біомакромолекул.

Ключові слова: парціальний молярний об'єм, вплив тиску, біомолекулярний розчин, теорія Кірквуда-Баффа, теорія RISM

PACS: 05.20.Jj, 05.70.Ce, 62.50.+p, 65.20.+w, 82.60.Lf, 87.15.Aa 
